BULL. AUSTRAL. MATH. SOC.

VOL. $4(1971), 205-209$.

\title{
Generalization of Hall planes of odd order
}

\author{
P. B. Kirkpatrick
}

\begin{abstract}
Some properties of projective planes having a certain type of collineation group are proved, and a class of these planes which properly contains the class of all hall plenes of odd order is explicitly constructed.
\end{abstract}

\section{Introduction}

The Hall plane satisfies the condition:

(1) $\Pi$ is a translation plane, and it has a Baer subplane $\pi_{0}$ fixed pointwise by a collineation group which is simply transitive on those points of the line at infinity which do not lie in $\Pi_{0}$. The line at infinity belongs to $\pi_{0}$.

We call planes satisfying (1) 'generalized Hall planes'. We will show (among other things) that when such a plane has odd or zero characteristic then the subplane $\Pi_{0}$ is desarguesian; and we will construct a class of these planes which appears, to the author, to include some new finite planes.

\section{Properties of generalized Hall planes}

Let $\Pi$ be a generalized Hall plane. Then $\Pi$ may be coordinatized by a (right distributive) V.-W. system $F$ which contains a subsystem $F_{0}$ corresponding to $\Pi_{0} \cdot \Pi_{0}$ is a translation plane since it contains 
the line at infinity of $\Pi$. We choose the coordinate quadrangle to lie in $\Pi_{0}$. )

We shall use Greek letters to denote elements of $F_{0}$.

THEOREM 1. If II is a plane of odd or zero characteristic satisfying (1), then $F_{0}$ is a skew field and $F$ is a right vector space of dimension 2 over $F_{0}$.

COROLLARY. $\Pi_{0}$ is desarguesian.

Proof of Theorem 1. Choose an element $z$ of $F \backslash F_{0}$. Let $w$ be any other element of $F$. Then, since $\Pi_{0}$ is a Baer subplane, the point $(z, w)$ lies on some line $y=x \alpha+\beta$ of $\Pi_{0}$, that is $w=z \alpha+\beta$ for some $\alpha, \beta \in F$.

The collineations fixing $\Pi_{0}$ pointwise correspond to automorphisms of $F$ which fix $F_{0}$ elementwise. So $(z \rho) \sigma=z \alpha+\beta$ for some $\alpha$ and $\beta$ which depend only on $\rho$ and $\sigma$. Also $((z+1) \rho) \sigma=(z+1) \alpha+\beta$, so $\alpha=\rho \sigma$ and $(z \rho) \sigma=z(\rho \sigma)+\beta$. Furthermore $((2 z) \rho) \sigma=(2 z)(\rho \sigma)+\beta$ and $2 \in \operatorname{Kern} F$, so $\beta=0$. Thus $(z \rho) \sigma=z(\rho \sigma)$ for all $\rho, \sigma \in F_{0}$. Similarly $z(\rho+\sigma)=z \rho+z \sigma$. [Start with $z \rho+z \sigma=z \lambda+\mu$. ]

For any $\rho, \sigma, \tau \in F_{0}$, we have:

$$
((z \rho) \sigma) \tau=(z \rho)(\sigma \tau)=z(\rho(\sigma \tau)),
$$

and

$$
((z \rho) \sigma) \tau=(z(\rho \sigma)) \tau=z((\rho \sigma) \tau) \text {. }
$$

Thus $\rho(\sigma \tau)=(\rho \sigma) \tau$; similarly $\rho(\sigma+\tau)=\rho \sigma+\rho \tau$. This completes the proof of the theorem.

From the multiplication operation in $F$ we obtain two mappings $f$ and $g$ of $F_{\mathrm{o}} \times F_{\mathrm{O}}$ onto $F_{\mathrm{O}}$, defined by:

$$
(z \alpha+\beta) z=z f(\alpha, \beta)+g(\alpha, \beta) .
$$

The V.-W. system $F$ may be described as follows: 
(2) $F$ is a right vector space of dimension 2 over a skew field $F_{0}$ embedded in it in the usual way, with a multiplication operation

$$
\begin{aligned}
& x . \alpha=x \alpha \text { (multiplication by a scalar) } \forall x \in F, \alpha \in F_{0}, \\
& (z \alpha+\beta) . z=z f(\alpha, \beta)+g(\alpha, \beta), \forall z \in F \backslash F_{0} ; \alpha, \beta \in F_{0},
\end{aligned}
$$

where $f$ and $g$ are mappings of $F_{0} \times F_{0}$ onto $F_{0}$.

The mappings $f$ and $g$ in (2) are of course not arbitrary.

THEOREM 2. A finite system $(F,+, \cdot)$ satisfying (2) is a V.-W. system if and only if

(a) $f$ and $g$ are additive homomorphisms with $f(0,1)=1$ and $g(0,1)=0$,

(b) for any given $\gamma$ and $\delta$, the equation $(f(\alpha, \beta), g(\alpha, \beta))=(\gamma, \delta)$ has exactly one solution $(\alpha, \beta)$, and

(c) the equation $(f(\alpha, \beta), g(\alpha, \beta))=(\alpha \gamma, \beta \gamma+\delta)$ has exactly one solution $(\alpha, \beta)$, given $\gamma$ and $\delta$; also, for this solution, $\alpha=0$ if and only if $\delta=0$.

Proof. The necessity of $(a)$ and $(b)$ follows immediately from the right distributivity of $F$ and the requirement that $\cdot$ be a loop operation on $E^{*}$. This loop requirement also implies (c). For consider the equation $z(z \alpha+\beta)=z \gamma+\delta$. Now, if $\alpha \neq 0$,

$$
\begin{aligned}
z(z \alpha+\beta) & =\left[(z \alpha+\beta)\left(\alpha^{-1}\right)-\beta \alpha^{-1}\right](z \alpha+\beta) \\
& =(z \alpha+\beta) f\left(\alpha^{-1},-\beta \alpha^{-1}\right)+g\left(\alpha^{-1},-\beta \alpha^{-1}\right) .
\end{aligned}
$$

If we replace $\alpha^{-1}$ by $\alpha$ and $-\beta \alpha^{-1}$ by $\beta$, the requirement that $z w=t$ has exactly one solution $w$ yields condition (c).

The sufficiency of $(a),(b),(c)$ is now evident, since, $F$ being finite, we merely need to show that these imply that $F$ is right distributive and that $\cdot$ is a loop operation on $F^{*}$.

A more complicated necessary and sufficient condition that $F$ be a (planar) V.-W. system is easily calculated for the case where $F$ is allowed to be infinite. 
We note that a V.-W. system satisfying (2) necessarily possesses a group of automorphisms which is transitive on $E \backslash F_{0}$ while fixing $F_{0}$ elementwise.

\section{A construction}

We start with an arbitrary finite field $F_{0}$ of odd order. Let $v$ be any non-square in $F_{0}$ and let $\theta$ and $\varphi$ be any two (possibly trivial, and possibly equal) automorphisms of $F_{0}$. We now construct a V.-W. system $(F,+, \cdot)$ from $F_{0}, \nu, \theta$ and $\varphi$.

Let $F$ be a right vector space of dimension 2 over $F_{0}$. Suppose $F_{0}$ is embedded in $F$ in the usual way. Addition is to be the same as vector addition, and multiplication to be given by the rules stated in (2) above, with the mappings $f$ and $g$ defined by:

$$
f(\alpha, \beta)=\beta^{\theta} \quad g(\alpha, \beta)=\alpha^{\varphi} \nu .
$$

Conditions (a), (b), (c) are easily verified, so that $(F,+, \cdot)$ is a V.-W. system. The plane $\pi$ over $F$ is a generalized Hall plane.

When $\theta=\varphi=1, F$ is the Hall system determined by $F_{0}$ and the polynomial $x^{2}-v$. Since Hall systems of the same order coordinatize isomorphic planes [5], the generalized Hall planes we have constructed include all Hall planes of odd order.

As in the Hall system for $F_{0}$ and $\nu$, we have for all $F$ : $z^{2}=\nu, \forall z \in F \backslash F_{0}$. But $\alpha z=z \alpha^{\theta}$ and $(z \alpha)_{z}=\alpha^{\varphi} \nu$ when $z \in F \backslash F_{0}$ and $\alpha \in F_{0}$.

In the case where $F_{0}=G F(9), \theta=1$ and $\varphi$ equals the non-trivial automorphism of $\mathrm{GF}(9)$, it is readily verified that $\mathrm{KernF}$ is the subfield of order 3 in GF(9). Since the Kern of any Hall system of order 81 is $G F(9)$, the plane over $F$ is not a Hall plane. By comparing the collineation group of the plane over $F$ with that of each of the Foulser generalized André planes of order 81 , it is not difficult 
to show that our class of planes is not a subclass of Foulser's: the Foulser planes of order 81 with Kern of order 3 all have a group of $10(X, O Y)$-homologies, whereas our plane has no such group (of order 10), no matter how $X$ and $Y$ be chosen on the line at infinity.

\section{References}

[1] P. Dembowski, Finite geometries (Ergebnisse der Mathematik und ihrer Grenzgebiete, Band 44, Springer-Verlag, Berlin, Heidelberg, New York, 1968).

[2] David A. Foulser, "A generalization of André's systems", Math. 2. 100 (1967), 380-395.

[3] Marshall Hall, "Projective planes", Trans. Amer. Math. Soc. 54 (1943), 229-277.

[4] Marshall Hall, Jr, The theory of groups (The Macmillan Company, New York, 1959).

[5] O.R. Hughes, "Collineation groups of non-desarguesian planes, I. The Hall-Veblen-Wedderburn systems", Amer. J. Math. 81 (1959), 921-938.

University of Sydney,

Sydney,

New South Wales. 